Cahiers d'études africaines

$236 \mid 2019$

Paroles de papier

\title{
COHEN Anouk. - Fabriquer le livre au Maroc
}

\section{Rémi Dewière}

\section{OpenEdition}

\section{Journals}

Édition électronique

URL : https://journals.openedition.org/etudesafricaines/28182

DOI : 10.4000/etudesafricaines.28182

ISSN : 1777-5353

Éditeur

Éditions de l'EHESS

\section{Édition imprimée}

Date de publication : 5 décembre 2019

Pagination : 1158-1161

ISBN : 9782713227813

ISSN : 0008-0055

Référence électronique

Rémi Dewière, " conen Anouk. - Fabriquer le livre au Maroc », Cahiers d'études africaines [En ligne], 236 2019, mis en ligne le 05 décembre 2019, consulté le 08 janvier 2022. URL : http:// journals.openedition.org/etudesafricaines/28182 ; DOI : https://doi.org/10.4000/etudesafricaines. 28182

Ce document a été généré automatiquement le 8 janvier 2022.

(c) Cahiers d'Études africaines 


\title{
COHEN Anouk. - Fabriquer le livre au
} Maroc

\author{
Rémi Dewière
}

\section{RÉFÉRENCE}

COHEN Anouk. - Fabriquer le livre au Maroc. Préface de Roger Chartier. Paris, Karthala (« IISMM »), 2016, 411 p., bibl., ill.

1 Centré sur l'objet livre, l'ouvrage d'Anouk Cohen tente de répondre à un paradoxe qui agite le Maroc contemporain: comment expliquer l'augmentation du nombre de maisons d'éditions dans le royaume, alors que l'ensemble de la profession évoque une crise du livre? Pour y répondre, l'auteure s'appuie sur une enquête de terrain minutieuse, ainsi que par une expérience professionnelle dans le milieu de l'édition et de la vente d'ouvrages étalée entre 2003 et 2010. Au Maroc, elle a travaillé dans une librairie et dans trois maisons d'éditions francophones, ainsi que dans une maison d'édition et librairie arabophone. L'une des particularités de son travail réside dans l'usage des travaux des historiens modernistes de l'écrit (p. 140) pour étudier le livre en tant qu'objet anthropologique (p. 38). Partant du présupposé que le processus d'alphabétisation du Maroc contemporain présente des similitudes avec l'Europe des $\mathrm{XVIII}^{\mathrm{e}}$ et $\mathrm{XIX}^{\mathrm{e}}$ siècles, elle mobilise avec pertinence les travaux des historiens de l'écrit à l'époque moderne, comme Adrian Johns, Christian Jacob ou Roger Chartier. Ce n'est pas un hasard si ce dernier a écrit la préface de l'ouvrage.

2 Il en ressort un travail novateur, très bien écrit et richement illustré. Le livre se construit autour du dualisme linguistique qui marque le monde de l'écrit au Maroc. Ces deux mondes sont alimentés par des mobilités humaines, des pratiques et des rapports sociaux-politiques radicalement différents, mais aussi des sons, des matérialités et des esthétiques distinctes. Anouk Cohen décrit alors une société plurilingue, où l'usage du français et de l'arabe sont «pour les lecteurs une marque de distinction et d'affirmation identitaire » (p. 32). Ainsi, elle remonte le fil de l'histoire du livre arabe et 
français, des espaces de lecture aux chambres des écrivains, en passant par les ateliers d'éditions. La première partie de son ouvrage étudie le livre, ses usages et les pratiques de lecture, des étals où ils sont vendus aux bibliothèques publiques et aux salons des particuliers. La confection du livre est au cœur de la deuxième partie de son ouvrage. Les passages concernant les librairies et maisons d'édition arabes du quartier des Habous de Casablanca font la richesse de cette partie, par le caractère inédit des informations recueillies. Enfin, la troisième partie est consacrée aux auteurs et au processus d'écriture, en plaçant au centre de l'analyse les auteurs et leur vécu. Cette approche à rebours vise à partir de ce qui est le plus visible pour arriver aux pratiques les plus intimes, ou cachées du livre : leur fabrication et leur écriture.

3 Fabriquer le livre au Maroc est une référence pour toute personne intéressée par l'écrit en Afrique. Contrairement à ce que son titre peut laisser entendre, l'ouvrage ne traite pas que de la fabrication du livre: les observations de l'auteure dans les salons privés, les bibliothèques publiques, les rues ou les imprimeries de Rabat et de Casablanca lui ont permis de travailler sur la "pluralité d'être » du livre (p. 18) et d'offrir un tableau exhaustif des pratiques d'écriture, d'édition et de lecture dans le Maroc urbain du début du XXI siècle. Anouk Cohen décline l'hétérogénéité des formes livresques, des livres familiaux (p. 327), aux livres pour enfants en passant par les ouvrages universitaires et les journaux. Cette volonté d'exhaustivité lui permet d'aborder la production de manuels scolaires (p.201), tirés à plusieurs dizaines de milliers d'exemplaires, tout comme les écrits brûlés ou intimes, qui échappent au procédé d'édition. Leur prise en compte lui permet d'aborder une question essentielle et souvent délaissée : le passage, parfois lourd de conséquences (p. 337), de l'écrit privé à l'écrit publié, de la parole honteuse (hchouma) à la parole censurée.

4 Le livre a également le mérite de prendre en considération des populations souvent mises en marge des études sur l'écrit : les femmes et les populations illettrées ou semilettrées. À ce titre, la description du choix du Coran par une femme dans une boutique est remarquable (pp. 116-118), tout comme le rôle des femmes dans les stratégies matrimoniales entre éditeurs et imprimeurs (p. 195) et les liens familiaux parmi les employées des ateliers d'édition (pp. 276-277). En particulier, l'auteure suit l'exemple de Nadia pour montrer l'impact des infographistes sur le rendu des livres édités au Maroc (pp. 250-253). Elle montre également comment les populations analphabètes ou semi-lettrées ont un rôle-clé dans la fabrication et la vente des livres. Leur rôle est notamment visible dans les chapitres 1 et 9, sur les vendeurs et les imprimeurs. En cela, son travail renvoie à celui d'Aïssatou Mbodj Pouye ou d'István György Tóth sur les sociétés à la lisière de l'alphabet. Ainsi, les systèmes de couleur de la couverture sont choisis à dessein par les éditeurs pour faciliter le travail des marchands de rue (pp. 57-59, 67), et leur choix en amont peut provoquer d'intenses discussions entre auteurs, éditeurs et illustrateurs (pp. 238-239).

Enfin, l'attention portée à la matérialité du livre est, à mon sens, le plus grand apport de ce travail. Le chapitre sur le Coran et de son étude en tant qu'objet, est particulièrement brillant. Les enjeux liés à sa fabrication (p. 105), à sa vente (p. 116) et à ses usages privés y sont décrits, en prêtant attention à sa position dans la maison, par exemple (p.122), où aux sens sollicités par l'objet, comme le parfum qui embaume les corans les plus récents (p. 124), ainsi qu'à la relation entre lecture et musicalité (p. 146). Ce chapitre a servi de point de départ à l'article de l'auteure dans ce numéro spécial. Un autre aspect bien traité concerne les pratiques de piratage du livre, dans un contexte 
où les acteurs du livre sont à la fois concurrents et doivent entretenir de bons rapports (p. 188), ou de la relation entre format du texte et censure du pouvoir (p. 308). Enfin, le chapitre 8 démontre de manière très convaincante que la mise en page d'un livre est à la foi marquée par les tensions entre les auteurs et les éditeurs, mais également entre des registres esthétiques et normatifs différents, et par le souci permanent de réaliser des économies (pp. 254-255).

Malgré la grande qualité de ce travail, on peut regretter que l'analyse manque, parfois, de nuance. L'opposition entre les valeurs de la francophonie et de l'arabophonie, souvent mise en exergue par certains acteurs du livre interrogés par Anouk Cohen, est parfois un peu trop marquée (pp. 232, 296). La description du rapport entre les écrivains arabisants avec leur langue (pp. 354-355) et l'évocation d'un marché « sous le manteau » du livre pornographique en langue arabe (p.373) auraient pu être mis en exergue pour relativiser cette opposition. Dans un autre registre, une meilleure connaissance de l'histoire des calligraphies arabes aurait donné une plus grande profondeur d'analyse à la partie sur l'édition des textes arabes (pp. 258-259). Toutefois, ces erreurs sont très anecdotiques pour un ouvrage qui affiche une connaissance du terrain et une finesse remarquables. Les scènes décrites sont des instantanés (l'auteure insiste à plusieurs reprises sur les mutations rapides du monde du livre au Maroc) qui permettent d'ouvrir plusieurs débats, astucieusement évoqués par l'auteure, à l'image de la question de la darîja (pp.300-304) et de l'institutionnalisation de la langue amazigh (p. 29, n. 12). De même, l'irruption d'internet et des réseaux sociaux mériterait de comparer le contexte marocain avec d'autres terrains, comme l'Europe. Enfin, le travail effectué sur les maisons d'édition de Casablanca et de Rabat en appelle un autre, tout aussi fascinant: celui des marges de l'écriture urbaine (les fanzines et revues alternatives), des mondes ruraux, des autres villes du Maroc, ou encore du Sahara Occidental.

7 Pour conclure, en affirmant que le monde du livre marocain est jeune, dualiste et urbain (p. 28), Anouk Cohen touche à des thématiques comme le rapport entre pouvoir et écrit, les évolutions de l'enseignement ou le rapport des sociétés maghrébines avec la langue. À de nombreuses reprises, le livre permet d'explorer des questions sociales plus larges encore, comme les évolutions des pratiques religieuses et de consommation (pp. 151-152), les années de plomb (p. 205), la marocanisation de l'écrit (à travers les contes pour enfants, les récits d'anciens détenus [p. 305]) ou encore le rapport avec l'extérieur (pp. 169-171). La phrase de Rachid, un des nombreux acteurs de ce livre, est très parlante en ce sens : le livre ne sert pas à « lire juste pour lire» (p. 141), mais il a une vocation plus globale dans une société marocaine en mutation. 